\title{
Oncicola venezuelensis (Marteau, 1977) (Acanthocephala: Oligacanthorhynchidae) in Puma concolor in Rio de Janeiro, Brazil
}

\author{
Oncicola venezuelensis (Marteau, 1977) (Acanthocephala: \\ Oligacanthorhynchidae) em Puma concolor no Rio de Janeiro, Brasil
}

\begin{abstract}
João Pedro Siqueira Palmer; Laís Verdan Dib¹,2; Lucas Fernandes Lobão1; Jessica Lima Pinheiro1; Raissa Cristina Ferreira Ramos ${ }^{1,2}$; Claudia Maria Antunes Uchoa'; Otilio Machado Pereira Bastos ${ }^{1}$; Maria Eduarda Monteiro Silva3; Jorge Luiz do Nascimento4; Alcides Pissinatti3,5; Alynne da Silva Barbosa ${ }^{1,2 \star}$ (D)

1 'Departamento de Microbiologia e Parasitologia, Instituto Biomédico, Universidade Federal Fluminense - UFF, Niterói, RJ, Brasil 2 Laboratório de Toxoplasmose e outras Protozooses, Instituto Oswaldo Cruz, Fundação Oswaldo Cruz - Fiocruz, Rio de Janeiro, RJ, Brasil ${ }^{3}$ Centro Universitário Serra dos Órgãos - Unifeso, Teresópolis, RJ, Brasil

${ }^{4}$ Parque Nacional da Serra dos Órgãos - Parnaso, Instituto Chico Mendes de Conservação de Biodiversidade - ICMBio, Teresópolis, RJ, Brasil ${ }^{5}$ Centro de Primatologia do Rio de Janeiro - CPRJ, Instituto Estadual do Ambiente - INEA, Guapimirim, RJ, Brasil
\end{abstract}

How to cite: Palmer JPS, Dib LV, Lobão LF, Pinheiro JL, Ramos RCF, Uchoa CMA, et al. Oncicola venezuelensis (Marteau, 1977) (Acanthocephala: Oligacanthorhynchidae) in Puma concolor in Rio de Janeiro, Brazil. Braz J Vet Parasitol 2020; 29 (3): e009620. https://doi.org/10.1590/S1984-29612020046

\begin{abstract}
Specimens of Oncicola venezuelensis (Marteau, 1977) were recovered from fragments of intestinal tissue of a female Puma concolar (Linn, 1771) found dead in Petrópolis, Rio de Janeiro in 2017. A total of 140 helminths were recovered. Five males and 5 females of the helminths were analyzed morphologically as well as 50 parasite eggs recovered in intestinal contents. Morphologically, these helminths were compatible with the genus Oncicola, because of the size and shape of the proboscis, the size and disposition of the lemnisci and the morphometry of the eggs, in which the external membrane of the shell was delicate and clear. From histopathology, the helminths were deeply embeded in the mucosa reaching up to the muscle layer. One specimen was also identified molecularly with universal primers that amplified the eukaryote region ITS1-5.8S-ITS2. The helminth showed $99 \%$ identity with the gene sequence of $O$. venezuelensis deposited in GenBank. It is important to emphasize, this parasite has been very little reported in the literature, which reinforces the importance of this report.
\end{abstract}

Keywords: Acanthocephala, Puma concolor, morphometry, molecular biology, histopathology.

\begin{abstract}
Resumo
Espécimes de Oncicola venezuelensis (Marteau, 1997) foram recuperados de fragmentos do tecido intestinal de uma fêmea de Puma concolor (Linn, 1771) encontrada morta em Petrópolis, Rio de Janeiro, em 2017. Um total de 140 helmintos foram recuperados. Cinco machos e 5 cinco fêmeas dos helmintos foram analisados morfologicamente, bem como 50 ovos dos parasitos recuperados no conteúdo intestinal. Morfologicamente, esses helmintos eram compatíveis com o gênero Oncicola, devido ao tamanho e formato da probóscide, o tamanho e disposição do leminisco e a morfometria dos ovos, que apresentaram membrana externa da casca delicada e clara. A partir da histopatologia, pode-se verificar que os helmintos estavam profundamente inseridos na mucosa, atingindo até a camada muscular. Um espécime também foi identificado molecularmente com primers universais que amplificam a região ITS-1.5.8S.ITS-2. Após as análises moleculares, foi verificado que os helmintos apresentavam $99 \%$ de identidade com sequência gênica de 0 . venezuelensis que está depositada no Genbank. É importante enfatizar, que esse parasito foi muito pouco relatado na literatura, demonstrando a importância deste relato.
\end{abstract}

Palavras-chave: Acanthocephala, Puma concolor, morfometria, biologia molecular, histopatologia.

Received April 23, 2020. Accepted May 8, 2020.

*Corresponding author: Alynne da Silva Barbosa. E-mail: alynnedsb@gmail.com

This is an Open Access article distributed under the terms of the Creative Commons Attribution License, which permits unrestricted use, distribution, and reproduction in any medium, provided the original work is properly cited. 


\section{Introduction}

There are around 1298 valid species of Acanthocephala distributed in four class (Amin, 2013). In Class Archiacanthocephala, Order Oligacanthorhynchida has a single Family Oligacanthorhynchidae, which contains 12 genera, including the genus Oncicola (Amin, 2013). This genus has 24 species that infect carnivorous animals such as mephetids, mustelids, procyonids, felids and canids (Amin, 2013). In the case of the genus Oncicola, carnivores are prominent definitive hosts (Nickol et al., 2006; Núñez \& Drago, 2017).

Helminths of the genus Oncicola inhabit the small intestine of the definitive hosts. The pathogenic condition that they cause relate mainly to the chronic inflammatory response that stems from their attachment to the host intestinal mucosa via their proboscis (Richardson, 2013; Núñez \& Drago, 2017). This may produce nodules similar to granulomas and, consequently, tissue fibrosis. In homeothermic hosts, the proboscis may penetrate deeply in the various layers of the small intestine (Núñez \& Drago, 2017).

Several species of Oncicola had already been described in Brazil, simply through morphological analyses of the adult forms. Among these species, O. sigmoides (Meyer, 1932) collected from Galictis sp. and Conepatus sp., and O. Iuehei (Travassos, 1917) from Nasua nasua (Linn., 1766), in Pará, São Paulo, Minas Gerais, Mato Grosso and Mato Grosso do Sul; O. macrurae (Meyer, 1931) from Leopardus wiedii (Schinz, 1821) in Pará; O. magalhaesi (Machado Filho, 1962) from Puma concolor (Linn., 1771) in São Paulo; O. micracantha (Machado Filho, 1949) from Conepatus chinga (Molina, 1782) in Rio Grande do Sul; O. paracampanulata (Machado Filho, 1963) from Puma yagouaroundi (Saint-Hilaire, 1803) in São Paulo, Paraná and Pará; O. oncicola (Ihering, 1892) from Panthera onca (Linn., 1758) in São Paulo and Minas Gerais, from P. yagouaroundi and Leopardus pardalis (Linn., 1758) in São Paulo and from L. wiedii in Pará; and O. campanulata (Diesing, 1851) from L. pardalis, Leopardus geoffroyi, P. onca and P. concolor, and O. chibigouzouensis (Machado Filho, 1963) from L. pardalis, in Mato Grosso (Vieira et al., 2008). The morphological description of $O$. venezuelensis form L. pardalis in the Serra da Capivara, Piauí was associated with molecular techniques once by Santos et al. (2017).

The aim of the present study was to report the occurrence of Oncicola venezuelensis infecting Puma concolor in the state of Rio de Janeiro, Brazil, using tools for microscopic, histopathological and molecular analysis.

\section{Material and Methods}

The result from the present analysis was inserted in a report on a larger project that was registered under SISBIO number 57635-3, authentication code 0576350320190522.

In August 2017, a female Puma concolor was found dead on the Brejal highway (Figure 1), in Petrópolis, state of Rio de Janeiro. The carcass was sent to the headquarters of the National Park of Serra dos Órgãos. The animal was of $1.2 \mathrm{~m}$ long and weighed $17 \mathrm{~kg}$.

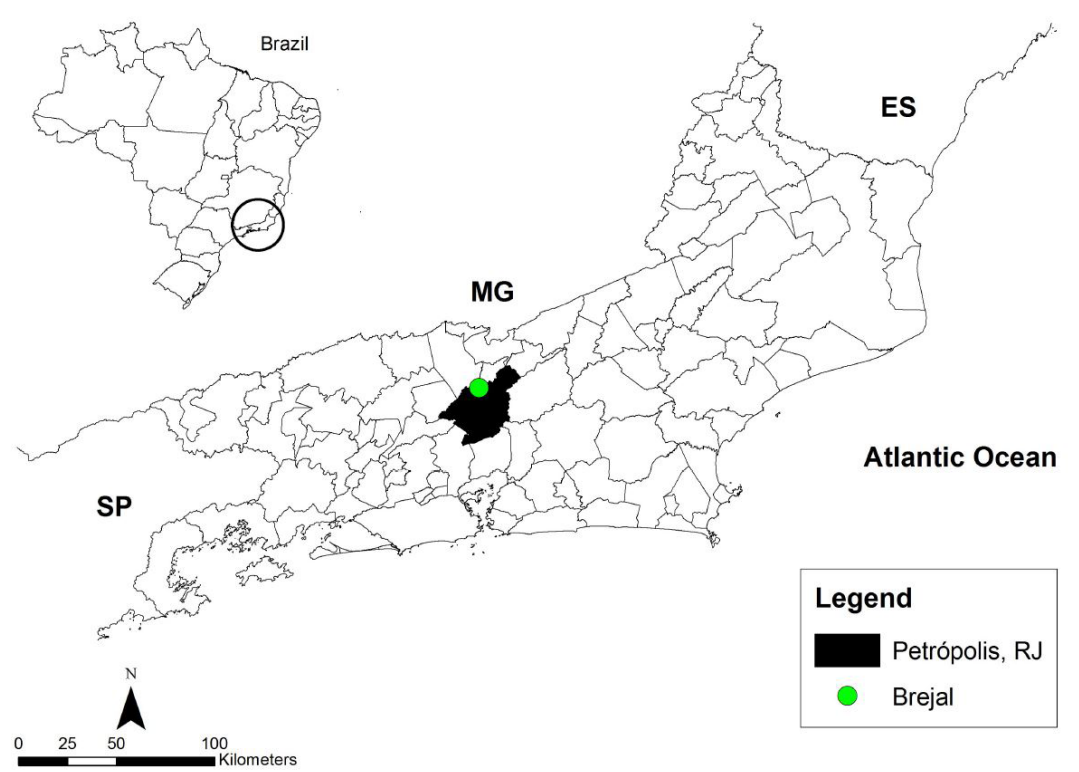

Figure 1. Location of the Brejal region in green and the city of Petrópolis in black, on a map of the state of Rio de Janeiro. 


\section{Morphology}

A segment of the small intestine was sent to the Parasitology Laboratory of the Fluminense Federal University. It was washed in a sterile buffered saline solution and the parasites adhering to the mucosa were carefully removed and were viewed under a stereoscopic microscope (Diag Tech ${ }^{\circledR}$ XTL 6445, São Paulo, Brazil). The helminths were stored in receptacles containing $70 \%$ glycerinated alcohol. Subsequently, they were cleared using $10 \%$ phenol for 30 minutes and were individually laid out on microscope slides for morphological analysis.

The liquid that resulted from washing the intestinal segment was aliquoted into conical-based tubes, for subsequent recovery of eggs. These tubes were subjected to centrifugation-sedimentation for 5 minutes, at $252 \mathrm{~g}$. Microscope slides of the sedimented eggs were covered with a $24 \times 32 \mathrm{~mm}$ coverslip.

Intestinal segments that contained the helminths were photographed. Parts of these tissues had been fixed in $10 \%$ formalin for 48 hours and then cut out, embedded in paraffin blocks and sectioned at $5 \mu \mathrm{m}$ using a microtome Leika RM 2125 RT (Leica ${ }^{\circledR}$, Germany) for mounting on microscope slides for analysis. These slides were stained with hematoxylin-eosin (hematoxylin - Confiança ${ }^{\circledR}$ São Paulo, Brazil; eosin - Reagen ${ }^{\circledast}$, Rio de Janeiro, Brazil).

The slides with the sectioned tissues were analyzed under an optical microscope. The adult forms of the helminths and the eggs were measured and photomicrographed under an optical microscope (Olympus ${ }^{\circledR}$ BX 41 , Tokyo, Japan) connected to a digital camera (Samsung ${ }^{\circledR}$ SDC415, Korea) using the software (Honestech ${ }^{\circledR}$ TVR, USA). Measurements of the helminths, their internal structures and their eggs were described using (minimum and maximum values), followed by the mean and standard deviation. So are these the specimens that were measured and included in Tables 1 and 2.

Table 1. Minimum and maximum values, means and standard deviations of the different parts of the bodies of female specimens of Oncicola and their eggs that were recovered in the present study and other studies.

\begin{tabular}{|c|c|c|c|c|}
\hline \multirow{3}{*}{ Biometry $(\mu \mathrm{m})$} & \multirow{3}{*}{$\begin{array}{c}\text { This study } \\
\text { Rio de Janeiro } \\
\text { Female }(n=5)\end{array}$} & \multicolumn{3}{|c|}{ Other reports about Oncicola sp. } \\
\hline & & $\begin{array}{c}\text { Oncicola venezuelensis } \\
\text { (Piauí. Brazil) } \\
\text { Santos et al. } 2017\end{array}$ & $\begin{array}{c}\text { Oncicola venezuelensis } \\
\text { (St. John Island. U. S. } \\
\text { Virgin Islands) Fuller, } \\
\text { Nickol. } 2011\end{array}$ & $\begin{array}{c}\text { Oncicola venezuelensis } \\
\text { (Venezuela) Marteu. } \\
1977\end{array}$ \\
\hline & & Female $(n=7)$ & Female $(n=15)$ & Female $(n=2)$ \\
\hline \multirow[t]{2}{*}{ Total lenght } & $6170-8520$ & $6250-13250$ & $13200-18300$ & $15000-16000$ \\
\hline & $7340 \pm 810$ & 9890 & 15500 & NR \\
\hline \multirow{2}{*}{ Total width } & $2010-2400$ & $1000-1950$ & $1800-2600$ & $1900-2200$ \\
\hline & $2200 \pm 120$ & 1470 & 2200 & NR \\
\hline \multirow[t]{2}{*}{ Proboscis lenght } & $500-550$ & $430-600$ & $432-480$ & $0.50-0.55$ \\
\hline & $520 \pm 24.49$ & 540 & 458 & NR \\
\hline \multirow[t]{2}{*}{ Proboscis width } & $550-600$ & $650-700$ & $528-566$ & $0.60-0.62$ \\
\hline & $602 \pm 34.87$ & 670 & 547 & NR \\
\hline \multirow[t]{2}{*}{ Proboscis receptacle } & $1300-1450$ & $970-1200$ & $1001-1390$ & $1200-1400$ \\
\hline & $1362.5 \pm 64.95$ & 1060 & 1210 & NR \\
\hline \multirow[t]{2}{*}{ Lemnisci } & $8600-13400$ & NR & $\leq 10000$ & $10000-12000$ \\
\hline & $10100 \pm 19000$ & NR & NR & NR \\
\hline \multicolumn{5}{|l|}{ Hooks } \\
\hline \multirow[t]{2}{*}{ I - Lenght } & $80-170$ & $110-140$ & 115-139 & $95-145$ \\
\hline & $107 \pm 23$ & 127 & 127 & NR \\
\hline \multirow[t]{2}{*}{ I - Base length } & $30-90$ & NR & NR & NR \\
\hline & $46.5 \pm 18.51$ & NR & NR & NR \\
\hline
\end{tabular}

NR: Not reported. 
Table 1. Continued...

\begin{tabular}{|c|c|c|c|c|}
\hline \multirow{3}{*}{ Biometry $(\mu \mathrm{m})$} & \multirow{3}{*}{$\begin{array}{c}\text { This study } \\
\text { Rio de Janeiro } \\
\text { Female }(n=5)\end{array}$} & \multicolumn{3}{|c|}{ Other reports about Oncicola sp. } \\
\hline & & $\begin{array}{c}\text { Oncicola venezuelensis } \\
\text { (Piauí. Brazil) } \\
\text { Santos et al. } 2017\end{array}$ & $\begin{array}{c}\text { Oncicola venezuelensis } \\
\text { (St. John Island. U. S. } \\
\text { Virgin Islands) Fuller, } \\
\text { Nickol. } 2011\end{array}$ & $\begin{array}{c}\text { Oncicola venezuelensis } \\
\text { (Venezuela) Marteu. } \\
1977\end{array}$ \\
\hline & & Female $(n=7)$ & Female $(n=15)$ & Female $(n=2)$ \\
\hline \multirow[t]{2}{*}{ II - Lenght } & $80-200$ & $80-120$ & $120-139$ & $95-135$ \\
\hline & $121 \pm 36.73$ & 103 & 131 & NR \\
\hline \multirow[t]{2}{*}{ II - Base length } & $30-110$ & NR & NR & NR \\
\hline & $73.5 \pm 23.51$ & NR & NR & NR \\
\hline \multirow[t]{2}{*}{ III - Lenght } & $70-180$ & $70-100$ & $98-110$ & $70-110$ \\
\hline & $106.5 \pm 24.75$ & 88 & 102 & NR \\
\hline \multirow[t]{2}{*}{ III - Base length } & $20-110$ & NR & NR & NR \\
\hline & $53.5 \pm 23.72$ & NR & NR & NR \\
\hline \multirow[t]{2}{*}{ IV - Lenght } & $70-190$ & $70-90$ & $91-96$ & $65-95$ \\
\hline & $115 \pm 23.45$ & 84 & 92 & NR \\
\hline \multirow[t]{2}{*}{ IV - Base length } & $20-60$ & NR & NR & NR \\
\hline & $36.25 \pm 10.53$ & NR & NR & NR \\
\hline \multirow[t]{2}{*}{ V - Lenght } & $50-130$ & $70-100$ & $77-86$ & $65-75$ \\
\hline & $94.5 \pm 16.87$ & 81 & 82 & NR \\
\hline \multirow[t]{2}{*}{$\mathrm{V}$ - Base length } & $20-60$ & NR & NR & NR \\
\hline & $33 \pm 9.54$ & NR & NR & NR \\
\hline \multirow[t]{2}{*}{ VI - Lenght } & $50-110$ & $50-70$ & $74-82$ & $60-75$ \\
\hline & $79.45 \pm 22.4$ & 62 & 77 & NR \\
\hline \multirow[t]{2}{*}{ VI- Base length } & $10-70$ & NR & NR & NR \\
\hline & $32.5 \pm 13.74$ & NR & NR & NR \\
\hline \multirow[t]{2}{*}{ Neck lenght } & $250-400$ & $300-340$ & $250-302$ & 500 \\
\hline & $314 \pm 48.86$ & 320 & 289 & NR \\
\hline \multirow[t]{2}{*}{ Neck width } & $496-504$ & $400-550$ & $384-422$ & 620 \\
\hline & $500 \pm 2.82$ & 476 & 396 & NR \\
\hline \multirow[t]{2}{*}{ Cerebral ganglion length } & $130-180$ & 110 & $110-149$ & NR \\
\hline & $152 \pm 17.88$ & NR & 131 & NR \\
\hline \multirow[t]{2}{*}{ Cerebral ganglion width } & $110-130$ & 110 & $62-86$ & NR \\
\hline & $112 \pm 10.95$ & NR & 73 & NR \\
\hline \multirow[t]{2}{*}{ Uterine bell length } & $300-650$ & $350-500$ & $499-538$ & 0.65 \\
\hline & $535 \pm 126.19$ & 422 & 520 & NR \\
\hline \multirow[t]{2}{*}{ Uterine bell width } & $103.6-150$ & NR & $259-336$ & 0.33 \\
\hline & $125.53 \pm 23.52$ & NR & 301 & NR \\
\hline
\end{tabular}

NR: Not reported. 
Table 1. Continued...

\begin{tabular}{|c|c|c|c|c|}
\hline \multirow{3}{*}{ Biometry $(\mu \mathrm{m})$} & \multirow{3}{*}{$\begin{array}{c}\text { This study } \\
\text { Rio de Janeiro } \\
\text { Female }(n=5)\end{array}$} & \multicolumn{3}{|c|}{ Other reports about Oncicola sp. } \\
\hline & & $\begin{array}{c}\text { Oncicola venezuelensis } \\
\text { (Piauí. Brazil) } \\
\text { Santos et al. } 2017\end{array}$ & $\begin{array}{c}\text { Oncicola venezuelensis } \\
\text { (St. John Island. U. S. } \\
\text { Virgin Islands) Fuller, } \\
\text { Nickol. } 2011\end{array}$ & $\begin{array}{c}\text { Oncicola venezuelensis } \\
\text { (Venezuela) Marteu. } \\
1977\end{array}$ \\
\hline & & Female $(n=7)$ & Female $(n=15)$ & Female $(n=2)$ \\
\hline \multirow[t]{2}{*}{ Uterus lenght } & $480-750$ & $400-720$ & $672-816$ & 1000 \\
\hline & $611.9 \pm 117.03$ & 610 & 752 & NR \\
\hline \multirow[t]{3}{*}{ Sphincter length } & $220-370$ & $200-400$ & $264-384$ & NR \\
\hline & $212 \pm 92.17$ & 318 & 330 & NR \\
\hline & eggs $(n=50)$ & eggs $(n=N R)$ & eggs (n=NR) & eggs (n=NR) \\
\hline \multirow[t]{2}{*}{ Length } & $35-74$ & $30-55$ & $67-72$ & NR \\
\hline & $59.8 \pm 11.29$ & 42 & 69 & \\
\hline \multirow[t]{2}{*}{ Width } & $25-70.3$ & $22-30$ & $43-50$ & NR \\
\hline & $39.4 \pm 10.12$ & 26 & 47 & \\
\hline
\end{tabular}

NR: Not reported.

\section{Molecular study}

To perform molecular analyses, five adult specimens, weighing-around $50 \mathrm{mg}$ were placed in a conical-based tube containing $15 \mathrm{~mL}$ of sterile buffered saline solution. This tube was then subjected to three cycles of centrifugation at $447 \mathrm{~g}$ for 10 minutes. Afterwards, the helminths were macerated on a Petri dish using a scalpel blade. The macerate was placed in a $1.5 \mathrm{~mL}$ microtube, $200 \mu \mathrm{L}$ of the tissue buffer and $40 \mu \mathrm{L}$ of the enzyme proteinase $\mathrm{K}$ was added. This material was then incubated at $37^{\circ} \mathrm{C}$ (Nova Técnica, São Paulo, Brazil) in a bacteriological chamber for 24 hours. Subsequenthly, $20 \mu \mathrm{L}$ of the enzyme proteinase $\mathrm{K}$ was added and incubate for 2 hours at $55^{\circ} \mathrm{C}$. After this incubation, no helminth tissue particles were evidenced. For DNA extraction the High Pure PCR Template Preparation (Roche ${ }^{\circledR}$, Indianapolis, USA) commercial kit was used.

The primer for implementing the PCR was chosen after verifying the morphological characteristics. A pair of universal eukaryote primers described by Chen et al. (2010) was used. These amplified the ITS1-5.8S-ITS2 region of RNAr: forward (5'-GTCGTAACAAGGTTTCCGTA -3') and reverse (5'-TATGCTTAARTTCAGCGGGT -3'). The total volume of the reaction mix was $25 \mu \mathrm{L}$, consisting of $5 \mu \mathrm{L}$ of each primer (at $10 \mathrm{pM}$ ), $7 \mu \mathrm{L}$ of the DNA extracted and $8 \mu \mathrm{L}$ of ultrapure water. For this PCR, the PuReTaq ${ }^{\mathrm{TM}}$ Ready-To-Go ${ }^{\mathrm{TM}}$ PCR (GE ${ }^{\circledR}$, New Jersey, USA) beads were used. The reaction was performed using the following cycling: $94^{\circ} \mathrm{C}$ for $2 \mathrm{~min}$, followed by 40 cycles at $95^{\circ} \mathrm{C}$ for $30 \mathrm{~s}, 55^{\circ} \mathrm{C}$ for $30 \mathrm{~s}, 72{ }^{\circ} \mathrm{C}$ for $60 \mathrm{~s}$ and $72{ }^{\circ} \mathrm{C}$ for $7 \mathrm{~min}$. Electrophoresis was performed using $1.5 \%$ agarose gel, and the bands were viewed by adding red gel. The amplified product was purified using the Promega commercial kit Wizard SV Gel and PCR Clean-Up System (Promega ${ }^{\circledR}$, Wisconsin, USA). Following this, the purified product was subjected to genetic sequencing in an automated sequencer. The sequences were input to the BioEdit 7.2.5 (Hall, 1999) software and were compared with the reference sequences deposited in GenBank (Table 3). To compile the phylogenetic tree, the Mega ${ }^{\circledR}$ software, version 6 (Tamura et al., 2013), with the maximum likelihood (ML) algorithm in the Tamura Nei model with 5000 bootstraps, was used.

\section{Results}

Morphology

\section{Oncicola venezuelensis (Marteau, 1977)}

A total of 140 helminths and 50 eggs were recovered. Measurements were made of 10 helminths ( 5 males and 5 females) and on all the eggs (Tables 1 and 2). Trunk globous that was slightly wider in the anterior portion. Males $6790 \mu \mathrm{m}$ long (5020-8210) by $2280 \mu \mathrm{m}$ wide (2060-2550) and females $7340 \mu \mathrm{m}$ long (6170-8520) and $2200 \mu \mathrm{m}$ 
Table 2. Minimum and maximum values, means and standard deviations of the different parts of the bodies of male specimens of Oncicola that were recovered in the present study and other studies.

\begin{tabular}{|c|c|c|c|c|}
\hline \multirow{3}{*}{ Biometry $(\mu \mathrm{m})$} & \multirow{3}{*}{$\begin{array}{c}\text { This study } \\
\text { Rio de Janeiro } \\
\text { Male }(n=5)\end{array}$} & \multicolumn{3}{|c|}{ Other reports about Oncicola sp. } \\
\hline & & $\begin{array}{c}\text { Oncicola venezuelensis } \\
\text { (Piauí. Brazil) } \\
\text { Santos et al. } 2017\end{array}$ & $\begin{array}{c}\text { Oncicola venezuelensis } \\
\text { (St. John Island. U. S. } \\
\text { Virgin Islands) Fuller, } \\
\text { Nickol } 2011\end{array}$ & $\begin{array}{c}\text { Oncicola venezuelensis } \\
\text { (Venezuela) Marteu. } \\
1977\end{array}$ \\
\hline & & Male $(n=3)$ & Male $(n=10)$ & Male $(n=2)$ \\
\hline \multirow[t]{2}{*}{ Total length } & $5020-8210$ & $5630-12500$ & $6500-8400$ & $13500-14000$ \\
\hline & $6790 \pm 970$ & 9170 & 8000 & NR \\
\hline \multirow[t]{2}{*}{ Total width } & $2060-2550$ & $750-1500$ & $1200-1300$ & 2000 \\
\hline & $2280 \pm 180$ & 1170 & 1200 & NR \\
\hline \multirow[t]{2}{*}{ Proboscis lenght } & $450-550$ & $400-500$ & $336-348$ & $0.50-0.55$ \\
\hline & $490 \pm 37.41$ & 433 & 344 & NR \\
\hline \multirow[t]{2}{*}{ Proboscis width } & $580-600$ & $370-600$ & $476-480$ & $0.60-0.62$ \\
\hline & $596 \pm 8$ & 507 & 479 & NR \\
\hline \multirow[t]{2}{*}{ Proboscis receptacle } & $1300-1400$ & $600-1180$ & $1001-1390$ & $1200-1400$ \\
\hline & $1334 \pm 76.31$ & 960 & 1210 & NR \\
\hline \multirow[t]{2}{*}{ Lemnisci } & $5500-13000$ & NR & $\leq 10000$ & $10000-12000$ \\
\hline & $9700 \pm 3400$ & NR & NR & NR \\
\hline \multicolumn{5}{|l|}{ Hooks } \\
\hline \multirow[t]{2}{*}{ I - Lenght } & $50-120$ & $120-125$ & $115-139$ & $95-145$ \\
\hline & $96.84 \pm 23.85$ & NR & 127 & NR \\
\hline \multirow[t]{2}{*}{ I - Base length } & $20-70$ & NR & NR & NR \\
\hline & $48.95 \pm 16.19$ & NR & NR & NR \\
\hline \multirow[t]{2}{*}{ II - Lenght } & $60-170$ & $145-150$ & $120-139$ & $95-135$ \\
\hline & $105.33 \pm 29.18$ & NR & 131 & NR \\
\hline \multirow[t]{2}{*}{ II - Base length } & $30-100$ & NR & NR & NR \\
\hline & $60.67 \pm 18.43$ & NR & NR & NR \\
\hline \multirow[t]{2}{*}{ III - Lenght } & $70-120$ & 125 & $98-110$ & $70-110$ \\
\hline & $97.5 \pm 14.36$ & NR & 102 & NR \\
\hline \multirow[t]{2}{*}{ III - Base length } & $20-70$ & NR & NR & NR \\
\hline & $38.13 \pm 11.03$ & NR & NR & NR \\
\hline \multirow[t]{2}{*}{ IV - Lenght } & $40-120$ & $105-110$ & $91-96$ & $65-95$ \\
\hline & $92.78 \pm 18.02$ & NR & 92 & NR \\
\hline \multirow[t]{2}{*}{ IV - Base length } & $20-70$ & NR & NR & NR \\
\hline & $37.22 \pm 11.16$ & NR & NR & NR \\
\hline \multirow[t]{2}{*}{ V - Lenght } & $70-120$ & $95-105$ & $77-86$ & $65-75$ \\
\hline & $90.56 \pm 16.05$ & NR & 82 & NR \\
\hline
\end{tabular}

NR: Not reported. 
Table 2. Continued...

\begin{tabular}{|c|c|c|c|c|}
\hline \multirow{3}{*}{ Biometry $(\mu \mathrm{m})$} & \multirow{3}{*}{$\begin{array}{c}\text { This study } \\
\text { Rio de Janeiro } \\
\text { Male }(n=5)\end{array}$} & \multicolumn{3}{|c|}{ Other reports about Oncicola sp. } \\
\hline & & $\begin{array}{c}\text { Oncicola venezuelensis } \\
\text { (Piauí. Brazil) } \\
\text { Santos et al. } 2017\end{array}$ & $\begin{array}{c}\text { Oncicola venezuelensis } \\
\text { (St. John Island. U. S. } \\
\text { Virgin Islands) Fuller, } \\
\text { Nickol } 2011\end{array}$ & $\begin{array}{c}\text { Oncicola venezuelensis } \\
\text { (Venezuela) Marteu. } \\
1977\end{array}$ \\
\hline & & Male $(n=3)$ & Male $(n=10)$ & Male $(n=2)$ \\
\hline \multirow[t]{2}{*}{ V - Base length } & $20-60$ & NR & NR & NR \\
\hline & $37.78 \pm 14.45$ & NR & NR & NR \\
\hline \multirow[t]{2}{*}{ VI - Lenght } & $50-110$ & $95-100$ & $74-82$ & $60-75$ \\
\hline & $92 \pm 16.57$ & NR & 77 & NR \\
\hline \multirow[t]{2}{*}{ VI- Base length } & $20-80$ & NR & NR & NR \\
\hline & $36 \pm 17.17$ & NR & NR & NR \\
\hline \multirow[t]{2}{*}{ Neck lenght } & $200-300$ & $220-270$ & $250-302$ & 500 \\
\hline & $254 \pm 40.7$ & 245 & 289 & NR \\
\hline \multirow[t]{2}{*}{ Neck width } & $200-550$ & 200 & $384-422$ & 620 \\
\hline & $424 \pm 122.08$ & NR & 396 & NR \\
\hline \multirow[t]{2}{*}{ Cerebral ganglion length } & $140-190$ & NR & $110-149$ & NR \\
\hline & $157.5 \pm 22.17$ & NR & 131 & NR \\
\hline \multirow[t]{2}{*}{ Cerebral ganglion width } & $100-140$ & NR & $62-86$ & NR \\
\hline & $124 \pm 17.07$ & NR & 73 & NR \\
\hline \multirow[t]{2}{*}{ Anterior testis length } & $500-660$ & $930-1180$ & NR & $1800-2000$ \\
\hline & $593.33 \pm 68.23$ & NR & NR & NR \\
\hline \multirow[t]{2}{*}{ Anterior testis width } & $370-550$ & $500-600$ & NR & 720 \\
\hline & $460 \pm 64.81$ & NR & NR & NR \\
\hline \multirow[t]{2}{*}{ Posterior testis length } & $540-730$ & $1040-1250$ & NR & NR \\
\hline & $608.33 \pm 66.99$ & NR & NR & NR \\
\hline \multirow[t]{2}{*}{ Posterior testis width } & $300-580$ & $600-680$ & NR & NR \\
\hline & $430 \pm 92.38$ & NR & NR & NR \\
\hline \multirow[t]{2}{*}{ Cement glands length } & $570-800$ & $600-700$ & $528-672$ & 850 \\
\hline & $712 \pm 77.04$ & NR & 595 & $N R$ \\
\hline \multirow[t]{2}{*}{ Cement glands width } & $420-950$ & $550-850$ & $269-288$ & 350 \\
\hline & $500 \pm 244.86$ & NR & 281 & NR \\
\hline \multirow[t]{2}{*}{ Safftigen's pouch length } & $600-790$ & $1150-1200$ & 1056-1392 & NR \\
\hline & $670 \pm 70.59$ & NR & 1264 & NR \\
\hline \multirow[t]{2}{*}{ Safftigen's pouch width } & $310-600$ & $550-650$ & $288-480$ & NR \\
\hline & $466 \pm 109.10$ & NR & 384 & NR \\
\hline \multirow[t]{2}{*}{ Copulatory bursa length } & $300-520$ & 1500 & NR & NR \\
\hline & $398.33 \pm 73.35$ & $N R$ & NR & NR \\
\hline
\end{tabular}

NR: Not reported. 
Table 2. Continued...

\begin{tabular}{|c|c|c|c|c|}
\hline \multirow{3}{*}{ Biometry $(\mu \mathrm{m})$} & This study & \multicolumn{3}{|c|}{ Other reports about Oncicola sp. } \\
\hline & Rio de Janeiro & $\begin{array}{c}\text { Oncicola venezuelensis } \\
\text { (Piauí. Brazil) } \\
\text { Santos et al. } 2017\end{array}$ & $\begin{array}{c}\text { Oncicola venezuelensis } \\
\text { (St. John Island. U. S. } \\
\text { Virgin Islands) Fuller, } \\
\text { Nickol } 2011\end{array}$ & $\begin{array}{c}\text { Oncicola venezuelensis } \\
\text { (Venezuela) Marteu. } \\
1977\end{array}$ \\
\hline & Male $(n=5)$ & Male $(n=3)$ & Male $(n=10)$ & Male $(n=2)$ \\
\hline \multirow[t]{2}{*}{ Copulatory bursa width } & $210-620$ & 550 & NR & NR \\
\hline & $356.67 \pm 131.23$ & NR & NR & NR \\
\hline
\end{tabular}

NR: Not reported.

Table 3. Helminth species and genera used in the phylogenetic analysis of this study.

\begin{tabular}{|c|c|c|c|}
\hline Species & Family & $\begin{array}{c}\text { Genbank acess } \\
\text { number }\end{array}$ & Genbank Reference \\
\hline Oncicola venezuelensis & Oligacanthorhynchidae & KU521566 & Santos et al. (2017) \\
\hline Oncicola sp. & Oligacanthorhynchidae & AF416416 & $\begin{array}{c}\text { Garcia-Varela et al. (2003) } \\
\text { Unpublished }\end{array}$ \\
\hline Macracanthorhynchus ingens & Oligacanthorhynchidae & AF416414.1 & $\begin{array}{c}\text { Garcia-Varela et al. (2003) } \\
\text { Unpublished }\end{array}$ \\
\hline Mediorhynchus sp. & Gigantorhynchydae & AF416413 & $\begin{array}{c}\text { Garcia-Varela et al. (2003) } \\
\text { Unpublished }\end{array}$ \\
\hline Acanthosentis cheni & Quadrigyridae & JX960752 & Song et al. (2013) Unpublished \\
\hline Neoechinorhynchus roseum & Neoechinorhynchidae & FJ388981 & Martínez-Aquino et al. (2009) \\
\hline Neoechinorhynchus emyditoides & Neoechinorhynchidae & KC004175 & $\begin{array}{l}\text { Pinacho-Pinacho et al. (2013) } \\
\text { Unpublished }\end{array}$ \\
\hline Neoechinorhynchus schmidti & Neoechinorhynchidae & KC004173 & $\begin{array}{l}\text { Pinacho-Pinacho et al. (2013) } \\
\text { Unpublished }\end{array}$ \\
\hline Pseudoacanthocephalus nguyenthileae & Echinorhynchidae & KC491890 & Tkach et al. (2013) \\
\hline Pseudoacanthocephalus nickoli & Echinorhynchidae & KC491884 & Tkach et al. (2013) \\
\hline Polymorphus minutus & Polymorphidae & AY532067 & Garcia-Varela et al. (2005) \\
\hline Polymorphus altmani & Polymorphidae & AY532066 & Garcia-Varela et al. (2005) \\
\hline Moniliformis moniliformis & Moniliformidae & AF416415 & $\begin{array}{c}\text { Garcia-Varela et al. (2003) } \\
\text { Unpublished }\end{array}$ \\
\hline Heteroxynema cucullatum & Heteroxynematidae & MH011309 & Bell et al., 2018 \\
\hline Necator americanus & Ancylostomatidae & MH6658431 & Monteiro et al. (2018) \\
\hline
\end{tabular}

wide (2010-2400). Proboscis globular, $490 \mu \mathrm{m}$ long (450-550) by $596 \mu \mathrm{m}$ wide (580-600) in males and $520 \mu \mathrm{m}$ long (500-520) and $602 \mu \mathrm{m}$ wide (550-600) in females with six rows of six hooks each. Proboscis receptacle single walled, $1334 \mu \mathrm{m}$ long (1300-1400) in males and $1362 \mu \mathrm{m}$ long (1300-1450) in females (Figure 2D). Cerebral ganglion, males $157.5 \mu \mathrm{m}$ long by $124 \mu \mathrm{m}$ wide and females $152 \mu \mathrm{m}$ long by $112 \mu \mathrm{m}$ wide. Neck short, males $254 \mu \mathrm{m}$ long (200-300) by $424 \mu \mathrm{m}$ wide (200-550) and females $314 \mu \mathrm{m}$ long (250-400) by 500 wide (496-504). Long tubular lemnisci reaching posterior portion of trunk, occasionally rolledinged up. Leminisci in the males had $9700 \mu \mathrm{m}$ long and in the females $10100 \mu \mathrm{m}$ long medium (Figure 2).

Males: Reproductive tract in posterior half of trunk. Testes elliptical, in tandem. Anterior testis $593 \mu \mathrm{m}$ long (500-600) by $460 \mu \mathrm{m}$ wide (370-550). Eight cement glands in pairs $712 \mu \mathrm{m}$ long (570-800) by $500 \mu \mathrm{m}$ wide (420-950) and Safftigen pouch $670 \mu \mathrm{m}$ long (600-790) by $466 \mu \mathrm{m}$ wide (310-600). Copulatory bursa $398 \mu \mathrm{m}$ long (300-520) and $356 \mu \mathrm{m}$ wide (210-620) (Figure 2A). 


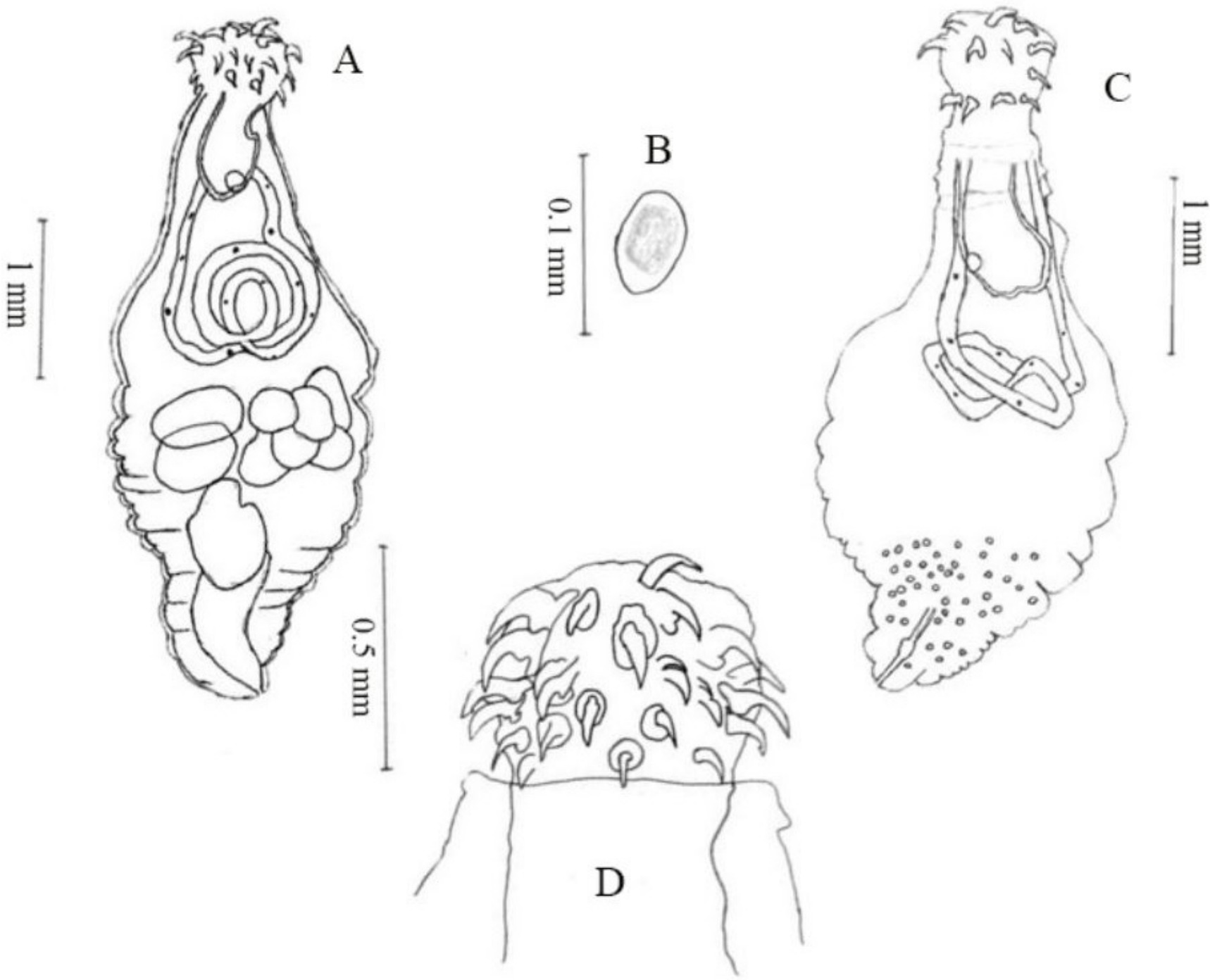

Figure 2. Line drawings of mature Oncicola venezuelensis from Puma concolor, in Rio de Janeiro. (A) Entire male; (B) Egg detected in sediment stool; (C) Outline showing shape of a gravid female; (D) Proboscis of a female.

Females: Uterine bell $535 \mu \mathrm{m}$ long (300-650) and $125.53 \mu \mathrm{m}$ wide (103.6-150), uterus $611 \mu \mathrm{m}$ long (480-750), uterine bell, vagina with sphincter $212 \mu \mathrm{m}$ long (220-370) and genital pore in the posterior region (Figure 2C). The eggs were generally very pale and slightly oval, with a very delicate external membrane with medium $59.8 \mu \mathrm{m}$ long by $39.4 \mu \mathrm{m}$ wide (Figure 2B). The measurements on the females, eggs and males are reported in Table 2 .

Macroscopically, adults partially attached to serous membrane intestinal wall could be seen and in mucous content (Figure 3A, B). Adults attached to the mucosa were surrounded by fibrous connective tissue (Figures 3C). Histopathological slides stained with hematoxylin-eosin showed that the parasites were attached by means of the proboscis and its hooks surrounded by collagenous tissue (Figures 3D, E). Parasite eggs are surrounded by host tissue (Figure 3F). Adult were deeply attached as far as the muscle layer (Figure 3G). The results from the morphological analysis, measurements on the parasites made it possible to identify them as members of the genus Oncicola.

\section{Molecular study}

After alignment of the nucleotide sequence obtained in the present study with other sequences of the phylum Acanthocephala retrieved from GenBank, it could be seen that the present sequence had $99 \%$ similarity with a sequence of Oncicola venezuelensis that had been recovered from a specimen of Leopardus pardalis in Serra da Capivara, Piauí (Figure 4). 

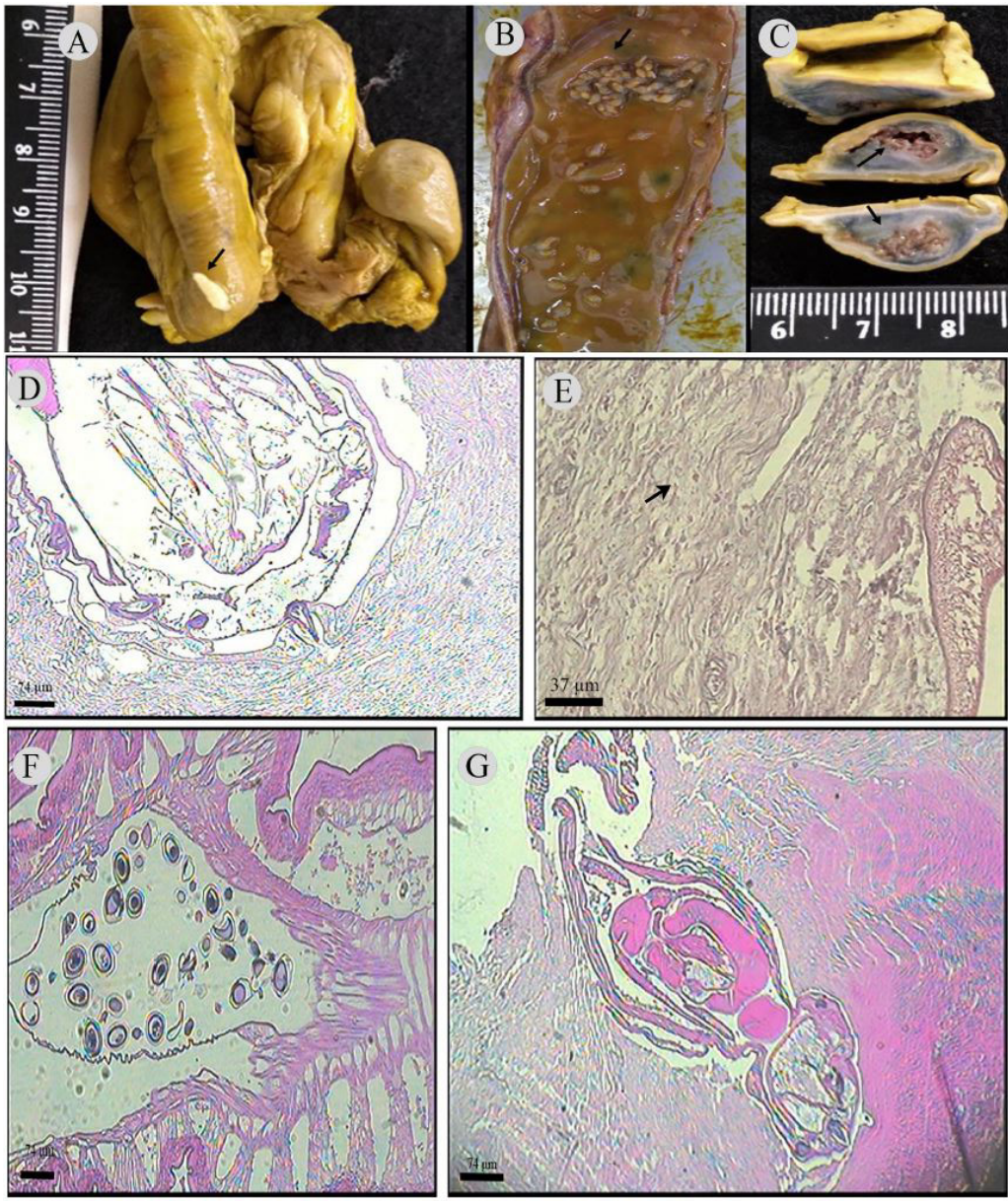

Figure 3. (A) Adult specimen attached to the serous membrane of the intestinal wall; (B) Segment jejunum with mucous content and specimens of adult attached to the mucosa; (C) Cut surface of a nodule located close to the pylorus region with parasites; (D) Histological section in which the proboscis with its hooks can be seen surrounded by collagenous tissue; (E). Collagenous tissue, in 400 x, observed in figure D; (F) Histological section through the nodule with parasite eggs surrounded by granuloma; (G) Histological section through a fragment of jejunum, in which the adult form of the parasite can be seen to be deeply attached to in muscle layer; (D-G) Hematoxylin and eosin staining.

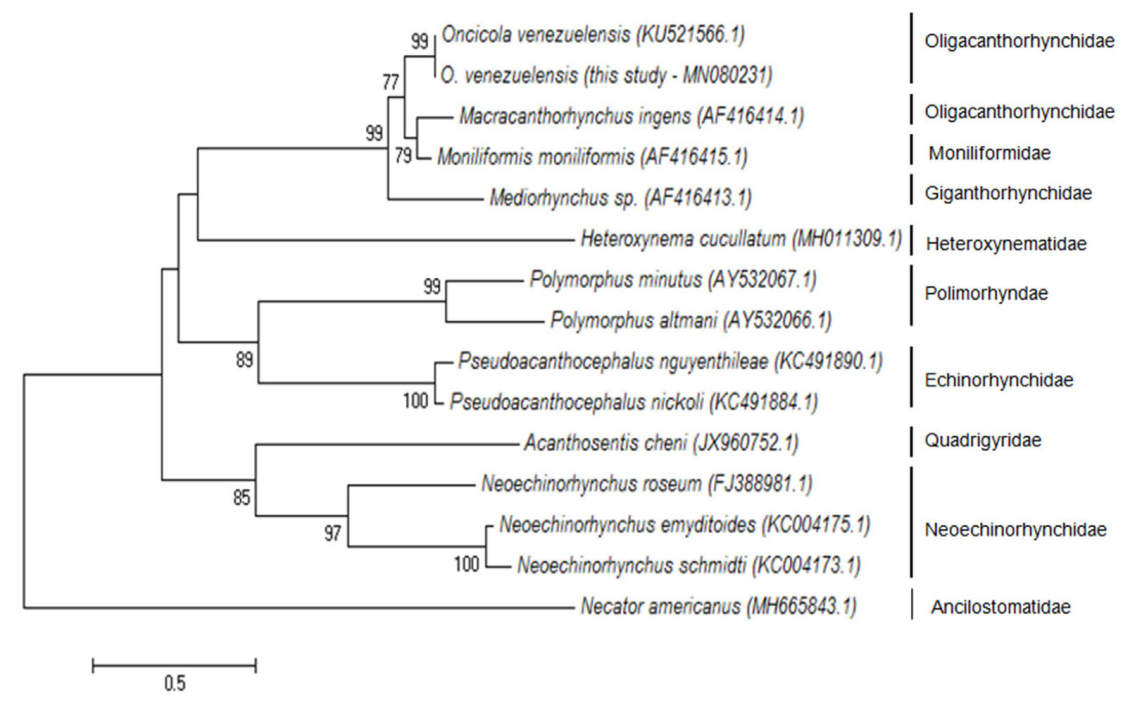

Figure 4. Maximum likelihood (ML) algorithm used with the Tamura Nei model that was based on the gene sequence obtained from the ITS1-5.8S-ITS2 region, compared with reference sequences from different species of helminths of the phylum Acanthocephala that had been detected in different animas, and from Necator americanus, obtained from GenBank. The numbers associated with the branches refer to the bootstrap values for 5000 replications. 


\section{Discussion}

In this study, it could be seen that the helminths presented morphology compatible with the family Oligacanthorhynchidae and genus Oncicola, since they had a globous body in the anterior region, a spherical proboscis with six rows of hooks, a proboscis receptacle on a single wall, inserted into the base of the proboscis, and presence of a cerebral ganglion. It was also observed that the males had cement glands. However, it was difficult to quantify the number of cement glands. This fact also reported by Yamaguti (1963). Oncicola hooks were strongly attached to the mucosa of the small intestine of the felid. This characteristic was similar to what was described by Richardson (2013) and Núñez \& Drago (2017). Through histopathology it was possible to confirm the proboscis' deep insertion in tissues, reaching the muscle layer. Unfortunately, through histopathology it was not possible to characterize the inflammatory cells in the most of the tissue, because of the autolysis process. The autolysis process occurred because the carcass was found at random in a Conservation Unit by a person, who had no experience in forensic analysis, so it is not known how long the carcass was exposed in the environment. This type of situation ends up being a reality evidenced in studies with wild animals in free living, highlighting the importance and rarity of the information that is generated with this type of material.

The eggs found in the present study had a delicate clear external shell membrane. This was morphologically similar to what was described by Yamaguti (1963) for the genus Oncicola and by Santos et al. (2017) and Fuller \& Nickol (2011) for the species O. venezuelensis, which were reported infecting, an ocelot in Piauí, Brazil, and a feral cat in the U.S. Virgin Islands, respectively.

In addition, tubular lemnisci were observed extending to the posterior trunk where they tapered and rolled up. Not much is known about the importance of lemnisci, but these structures may have a function relating to transportation of fluids to the proboscis, with importance in the hydraulic system for its eversion (Núñez \& Drago, 2017). In the present study, the lemnisci were long: around $9700 \mu \mathrm{m}$ in males and $10000 \mu \mathrm{m}$ in females. They occupied a large portion of the parasite's body. Fuller \& Nickol (2011) also reported similar measurement for lemnisci in O. venezuelensis, of around $10000 \mu \mathrm{m}$.

According to Marteau (1977), few authors have reported measurements on lemnisci in species of the genus Oncicola. Nonetheless, this structure is fundamental in microscopy for classifying this parasite. This author provided the first description of $O$. venezuelensis infecting $L$. pardalis in Venezuela and also reported characteristics of the lemnisci that were similar to what was found in the present study, with compatible length measurements of around 10000 to $12000 \mu \mathrm{m}$.

Measurements of the proboscis were compatible with described by Santos et al. (2017) and Marteau (1977), who reported that the proboscis was around 500 to $550 \mu \mathrm{m}$ in length by 600 to $620 \mu \mathrm{m}$ in width. However, measurements the proboscis of specimens from wild felids in Brazil, both in Rio de Janeiro and in Piauí, as well as from an ocelot in Venezuela, were slightly bigger than those from worms infecting feral cats in the United States by Fuller \& Nickol (2011). Changes in biometry of worms may be related to geographical locations and hosts species (Barbosa et al., 2017). Morphological examination of our specimens suggested that they might belong to the species $O$. venezuelensis. In order to ensure correct identification and study the phylogenetic interrelationships of these specimens we performed a molecular analysis using universal eukaryote primers that amplified the ITS1-5.8S-ITS2 region (Chen et al., 2010). In general, the sequence analyzed was within the Acanthocephala group next to Macracanthorhynchus, a genus that is also inserted into the same Oncicola family, Oligacanthorhynchidae. The sequence analyzed showed $99 \%$ identity with the species 0 . venezuelensis. The high branch support in the phylogenetic tree as well as the pairwise comparison evidence confirmed the identity of our specimens as O. venezuelensis.

The histopathological analysis on the tissues showed that the parasites were strongly attached to the deep tissue layers of the small intestine. In some places, they were surrounded by collagenous tissue, thus suggesting chronic infection. Despite the strong insertion of the parasite in the host tissue with the proboscis, in this report it was not possible to characterize the inflammatory cells, since the tissue was already very autolyzed, since the feline carcass was found in the environment for an undetermined period of the time. Although this finding is rare, Núñez \& Drago (2017) emphasized that when high parasite loads are present, acanthocephalans may cross the intestinal wall, thus causing the death of the host, since they carry bacteria that can reach the peritoneal cavity.

This is the second report of $O$. venezuelensis in Brazil. However, this is the first description of this infection in $P$. concolor. It is important to emphasize that the number of nucleotide sequences in the phylum Acanthocephala deposited in GenBank remains very small. This is especially so regarding the genus Oncicola, which draws attention to the need to conduct molecular studies to phylogenetically validate the 24 species of Oncicola, which have only been reported through morphological descriptions. 


\section{Acknowledgements}

We would like to thank the National Park of Serra dos Órgãos, the University Center of Serra dos Órgãos and the Primatology Center of the State of Rio de Janeiro for supplying the helminths and tissues, which were fundamental for conducting this study.

\section{References}

Amin OM. Classification of the Acanthocephala. Folia Parasitol 2013; 60(4): 273-305. http://dx.doi.org/10.14411/fp.2013.031. PMid:24261131.

Barbosa AS, Dib LV, Uchôa CMA, Bastos OMP, Pissinatti A. Trypanoxyuris (Trypanoxyuris) minutus (Schneider, 1866) among Alouatta guariba clamitans (Cabrera, 1940) in the state of Rio de Janeiro, Brazil. J Med Primatol 2017; 46(3): 101-105. http://dx.doi. org/10.1111/jmp.12265. PMid:28349584.

Bell KC, Demboski JR, Cook JA. Sympatric parasites have similar host-associated, but asynchronous, patterns of diversification. Am Nat 2018; 192(3): E106-E119. http://dx.doi.org/10.1086/698300. PMid:30125233.

Chen MX, Zhang LQ, Wen CG, Sun J, Gao Q. Phylogenetic relationship of species in the genus Aspidogaster (Aspidogastridae, Aspidogastrinae) in China as inferred from its rDNA sequences. Shui Sheng Sheng Wu Hsueh Bao 2010; 34(2): 312-316. http:// dx.doi.org/10.3724/SP.J.1035.2009.00312.

Fuller CA, Nickol BB. A Description of Mature Oncicola venezuelensis (Acanthocephala: Oligacanthorhynchidae) from a feral house cat in the U.S. Virgin Islands.J Parasito/ 2011; 97(6): 1099-1100. http://dx.doi.org/10.1645/GE-2849.1. PMid:21671723.

García-Varela M, Aznar FJ, Pérez-Ponce de León G, Piñero D, Laclette JP. Molecular phylogeny of Corynosoma Lühe, 1904 (Acanthocephala), based on 5.8S and internal transcribed spacer sequences. J Parasitol 2005; 91(2): 345-352. http://dx.doi. org/10.1645/GE-3272. PMid:15986610.

Garcia-Varela M, Cummings MP, Laclette JP. Phylogenetic Relationships of Archiacanthocephala (Acanthocephala) based on Gene Sequences of 16S, 5.85 and 185 rRNA, Internal Transcribed Spacers 1 and 2, and COI. Direct Submission Genbank; 2003. AF416416; AF416414.1; AF416413 (submission numbers).

Hall TA. BioEdit: a user-friendly biological sequence alignment editor and analysis program for Windows 95/98/NT. Nucleic Acids Symp Ser 1999; 41: 95-98.

Marteau M. Oncicola venezuelensis n. sp. (Archiacanthocephala; Oligacanthorhynchida) parasite de l'Ocelot (Felis pardalis L.). Ann Parasitol Hum Comp 1977; 52(1): 25-33. http://dx.doi.org/10.1051/parasite/1977521025. PMid:900772.

Martínez-Aquino A, Reyna-Fabián ME, Rosas-Valdez R, Razo-Mendivil U, Pérez-Ponce de León G, García-Varela M. Detecting a complex of cryptic species within Neoechinorhynchus golvani (Acanthocephala: Neoechinorhynchidae) inferred from ITSs and LSU rDNA gene sequences. J Parasitol 2009; 95(5): 1040-1047. http://dx.doi.org/10.1645/GE-1926.1. PMid:19438288.

Monteiro KJL, Calegar DA, Carvalho-Costa FA, Jaeger LH. Kato-Katz thick smears as a DNA source of soil-transmited helminths. J Helminthol 2018; 94: e10. http://dx.doi.org/10.1017/S0022149X18001013. PMid:30428936.

Nickol BB, Fuller CA, Rock P. Cystacanths of Oncicola venezuelensis (Acanthocephala: Oligacanthorhynchidae) in Caribbean termites and various paratenic hosts in the U.S. Virgin Islands. J Parasitol 2006; 92(3): 539-542. http://dx.doi.org/10.1645/GE3557.1. PMid:16883997.

Núñez V, Drago F. Phylum Acanthocephala. In: Drago FB, editor. Macroparásitos: diversidad y biologia. La Plata: Universidad Nacional de la Plata; 2017. p. 112-127.

Pinacho-Pinacho CD, Sereno-Uribe AL, Garcia-Varela M. Molecular and morphological data reveal a new species of Neoechinorhynchus (Acanthocephala: Neoechinorhynchidae) from Dormitator maculatus in the Gulf of Mexico. Direct Submission Genbank; 2013. KC004175 - KC004173 (submission number).

Richardson DJ. Achanthocepha. In: Wiley InterScience, editor. Encyclopedia of life sciences. Chichester: John Wiley \& Sons; 2013.

Santos EGN, Chame M, Chagas-Moutinho VA, Santos CP. Morphology and molecular analysis of Oncicola venezuelensis (Acanthocephala: Oligacanthorhyncidae) from the ocelot Leopardus pardalis in Brazil. J Helminthol 2017; 91(5): 605-612. http:// dx.doi.org/10.1017/S0022149X16000651. PMid:27669886.

Song, R, Li W, Wu S, Zou H, Wang, G. Population genetic structure of the acanthocephalan Acanthosentis cheni in the anadromous, freshwater, and landlocked stock of Coilia nasus: evolutionary adaption of marine helminth to freshwater. Direct Submission Genbank; 2013. JX960752 (submission number).

Tamura K, Stecher G, Peterson D, Filipski A, Kumar S. MEGA6: Molecular Evolutionary Genetics Analysis version 6.0. Mol Biol Evol 2013; 30(12): 2725-2729. http://dx.doi.org/10.1093/molbev/mst197. PMid:24132122. 
Tkach VV, Lisitsyna OI, Crossley JL, Binh TT, Bush SE. Morphological and molecular differentiation of two new species of Pseudoacanthocephalus Petrochenko, 1958 (Acanthocephala: Echinorhynchidae) from amphibians and reptiles in the Philippines, with identification key for the genus. Syst Parasito/ 2013; 85(1): 11-26. http://dx.doi.org/10.1007/s11230-013-9409-8. PMid:23595488.

Vieira FM, Luque JL, Muniz-Pereira LC. Checklist of helminth parasites in wild carnivore mammals from Brazil. Zootaxa 2008; 1721(1): 1-23. http://dx.doi.org/10.11646/zootaxa.1721.1.1.

Yamaguti S. Systema Helminthum. New York: Interscience Publishers; 1963. (vol. V, The Acanthocephala of Vertebrates). 